\title{
Spin-Electric Coupling in Molecular Magnets
}

\author{
Mircea Trif, ${ }^{1}$ Filippo Troiani, ${ }^{2}$ Dimitrije Stepanenko, ${ }^{1}$ and Daniel Loss ${ }^{1}$ \\ ${ }^{1}$ Department of Physics, University of Basel, Klingelbergstrasse 82, CH-4056 Basel, Switzerland \\ ${ }^{2}$ CNR-INFM National Research Center S3 c/o Dipartimento di Fisica via G. Campi 213/A, 41100, Modena, Italy
}

(Dated: October 31, 2018)

\begin{abstract}
We study the triangular antiferromagnet $\mathrm{Cu}_{3}$ in external electric fields, using symmetry group arguments and a Hubbard model approach. We identify a spin-electric coupling caused by an interplay between spin exchange, spin-orbit interaction, and the chirality of the underlying spin texture of the molecular magnet. This coupling allows for the electric control of the spin (qubit) states, e.g. by using an STM tip or a microwave cavity. We propose an experimental test for identifying molecular magnets exhibiting spin-electric effects.
\end{abstract}

PACS numbers: 75.50.Xx, 03.67.Lx

Single-molecule magnets (SMMs) 1] have emerged as a fertile testing ground for investigating quantum effects at the nanoscale, such as tunneling of magnetization [2, 3, 4], or coherent charge transport [5, 6, 7], or the decoherence and the transition from quantum to classical behavior 8]. SMMs with antiferromagnetic coupling between neighboring spins are especially promising for the encoding and manipulation of quantum information [9, 10, 11, 12], for they act as effective two-level systems, while providing additional auxiliary states that can be exploited for performing quantum gates, even in the presence of untunable couplings between the qubits 13]. Intra- and inter-molecular couplings of SMMs can be engineered by molecular and supra-molecular chemistry [14], enabling a bottom-up design of molecule-based devices [15].

While the properties of SMMs can be modfied during the synthesis, control on the time scales required for quantum information processing remains a challenge. The standard spin-control technique is electron spin resonance (ESR) driven by ac magnetic fields $B_{a c}(t)$ [8, 16, 17, 18]. For manipulation on the time scale of 1 ns (Rabi frequency $\Omega_{R} \sim 10^{9} \mathrm{~s}^{-1}$ ) $B_{a c}$ should be of the order of $10^{-2} \mathrm{~T}$, which, however, is difficult to achieve. The spatial resolution of $1 \mathrm{~nm}$, required for addressing a single molecule, is also prohibitively small. At these spatial and temporal scales, the electric control is preferable, because strong electric fields can be applied to small regions by using, for example, STM tips [19, 20, 21]. Also, the quantized electric field inside a microwave cavity can be used [22, 23, 24, 25] to control single qubits and to induce coupling between them even if they are far apart.

Here we identify and study an efficient spin-electric coupling mechanism in SMMs which is based on an interplay of spin exchange, spin-orbit interaction (SOI), and lack of inversion symmetry. Spin-electric effects induced solely by SOI [26] have been proposed [27] and experimentally demonstrated [28] in quantum dots. However, these SOI effects scale with the system size $L$ as $L^{3}$ [27], making them irrelevant for the much smaller SMMs. Thus, additional ingredients-such as broken symmetries- must be present in SMMs for an efficient coupling between spin and applied electric field.

In the following, we demonstrate the possibility of such spin-electric effects in SMMs by focusing on a specific example, namely an equilateral spin triangle, $\mathrm{Cu}_{3}$ [29]. In this SMM, the low energy states exhibit a chiral spin texture and, due to the absence of inversion symmetry, electric fields couple states of opposite chirality. Moreover, SOI couples the chirality to the total spin, and thus an effective spin-electric interaction eventually emerges.

Spin-electric coupling. The low-energy states of $\mathrm{Cu}_{3}$ can be described in terms of an effective spin Hamiltonian. There, three spins- $1 / 2 \mathbf{s}_{i}$ (one for each $\mathrm{Cu}^{2+}$ ion) are coupled through Heisenberg and DzyaloshinskiMoriya interaction, accounting for (super-) exchange and spin orbit interaction, resp. [29],

$$
H_{0}=\sum_{i=1}^{3} J_{i+1} \mathbf{s}_{i} \cdot \mathbf{s}_{i+1}+\sum_{i=1}^{3} \mathbf{D}_{i i+1} \cdot \mathbf{s}_{i} \times \mathbf{s}_{i+1} .
$$

The $\mathrm{D}_{3 h}$ symmetry of the triangle implies several relations between the coupling constants [30]. Since $J_{p q} \sim$ $5 \mathrm{~K}$ and $\left|\mathbf{D}_{p q}\right| \sim 0.5 \mathrm{~K}$, the Heisenberg terms determine the gross structure of the energy spectrum, and the Dzyaloshinski-Moriya terms the fine one. In particular, due to the antiferromagnetic coupling $\left(J_{p q}>0\right)$, the ground state multiplet corresponds to total spin $S=1 / 2$, and the gap to the first excited $S=3 / 2$ quadruplet is $\Delta_{\mathrm{H}} \equiv 3 J / 2$. The $S=1 / 2$ subspace is spanned by the symmetry-adapted states $|\chi, M\rangle$, i.e.,

$$
\begin{aligned}
& | \pm 1,+1 / 2\rangle \equiv\left(|\downarrow \uparrow \uparrow\rangle+\epsilon_{ \pm}|\uparrow \downarrow \uparrow\rangle+\epsilon_{\mp}|\uparrow \uparrow \downarrow\rangle\right) / \sqrt{3}, \\
& | \pm 1,-1 / 2\rangle \equiv\left(|\uparrow \downarrow \downarrow\rangle+\epsilon_{ \pm}|\downarrow \uparrow \downarrow\rangle+\epsilon_{\mp}|\downarrow \downarrow \uparrow\rangle\right) / \sqrt{3},
\end{aligned}
$$

with $\epsilon_{ \pm}=\mathrm{e}^{ \pm i 2 \pi / 3}$, that are simultaneous eigenstates of the chirality operator $\mathcal{C}_{z}$ and of $S_{z}$ (total spin), for the respective eigenvalues $\chi$ and $M$. Here, we have introduced the chirality vector $\mathcal{C}$ with components

$$
\begin{aligned}
& \mathcal{C}_{x}=(-2 / 3)\left(\mathbf{s}_{1} \cdot \mathbf{s}_{2}-2 \mathbf{s}_{2} \cdot \mathbf{s}_{3}+\mathbf{s}_{3} \cdot \mathbf{s}_{1}\right), \\
& \mathcal{C}_{y}=(2 / \sqrt{3})\left(\mathbf{s}_{1} \cdot \mathbf{s}_{2}-\mathbf{s}_{3} \cdot \mathbf{s}_{1}\right), \\
& \mathcal{C}_{z}=(4 / \sqrt{3}) \mathbf{s}_{1} \cdot\left(\mathbf{s}_{2} \times \mathbf{s}_{3}\right) .
\end{aligned}
$$



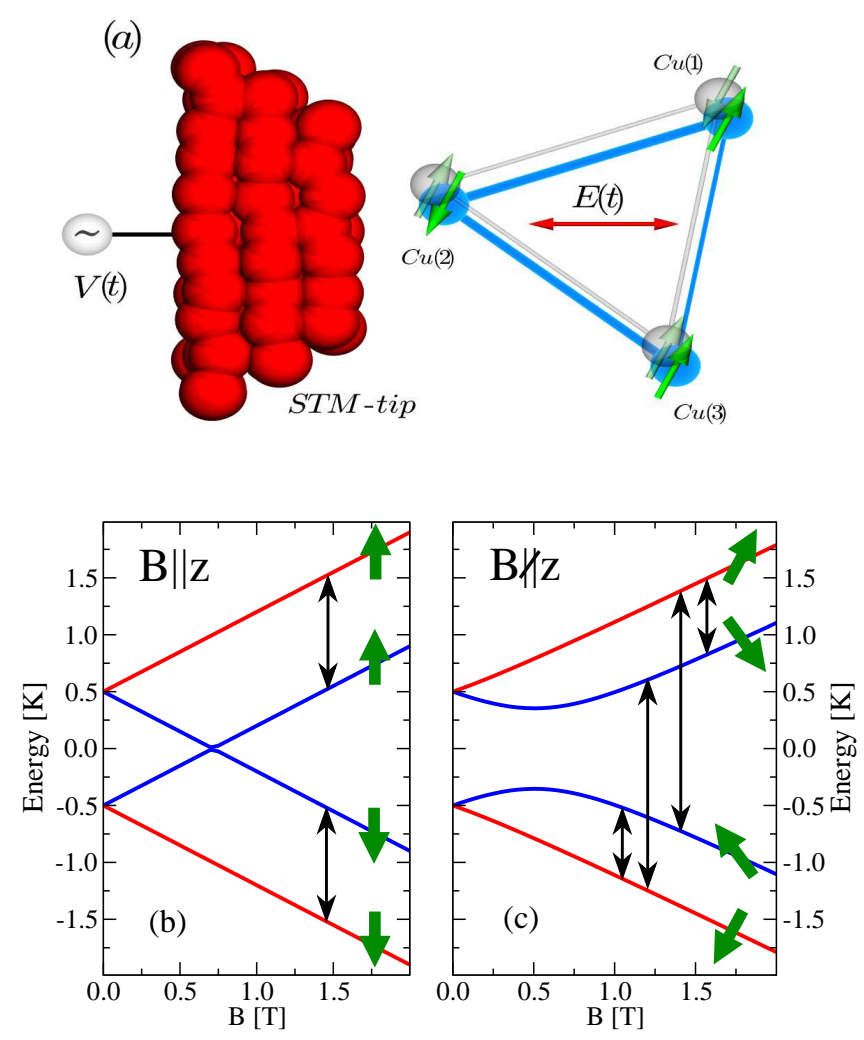

FIG. 1: (Color online) (a) $\mathrm{Cu}_{3}$-triangle exposed to an electric field $\mathbf{E}$ created by, e.g., an STM-tip. For $\mathbf{E}=0$, the exchange couplings in the molecule are equal (light triangle). A finite $\mathbf{E}$ affects the (super-) exchange coupling in a directional way (dark triangle). (b, c) Low-energy $S=1 / 2$ states of $\mathrm{Cu}_{3}$ in a magnetic field $\mathbf{B}$, with the zero-field SOI splitting $\Delta_{\mathrm{SO}}=1 \mathrm{~K}$. Light (red) and dark (blue) lines correspond to states with $\chi=+1(-1)$. If $\mathbf{B} \| z(\mathrm{~b})$, the transitions induced by $\mathbf{E}$ (thin arrows) conserve $S_{z}$; for $\mathbf{B} \nVdash z$ (c), these transitions result in a change of spin orientation (thick arrows).

They satisfy $\left[\mathcal{C}_{k}, \mathcal{C}_{l}\right]=i 2 \epsilon_{k l n} \mathcal{C}_{n}$ and $\left[\mathcal{C}_{k}, S_{l}\right]=0$, and act as Pauli matrices in the $|\chi= \pm 1\rangle$ bases. The states $|\chi, M\rangle$ are split by the SOI (see below) into two Kramers doublets, $| \pm 1, \pm 1 / 2\rangle$ and $| \pm 1, \mp 1 / 2\rangle$.

Next, we study the effect of an electric field $\mathbf{E}$ on the $\mathrm{Cu}_{3}$-spins using general symmetry group arguments. The low-energy molecular states form ${ }^{2} E^{\prime}$ and ${ }^{4} A_{2}^{\prime}$ irreducible representations (IRs) of $\mathrm{D}_{3 h}$, with the $S=$ $1 / 2$-states lowest in energy and transforming as two $E^{\prime}$ IRs, while the higher energy $S=3 / 2$-states span the four $A_{2}^{\prime}$ IRs [29, 30]. The chiral states, Eqs. (213), form the standard bases of the $E^{\prime}$-representations, with $\left|E_{ \pm}^{\prime}, S_{z}\right\rangle=\left|\chi= \pm 1, S_{z}\right\rangle$. An electric field $\mathbf{E}$ couples to $\mathrm{Cu}_{3}$ via $e \mathbf{E} \cdot \mathbf{R}$, where $e$ is the electron charge, and $\mathbf{R}=\sum_{j=1}^{3} \mathbf{r}_{j}$ transforms according to the reducible vector representation $D_{\mathrm{v}}=A_{2}^{\prime} \oplus E^{\prime}$. The $A_{2}^{\prime}$ IR acts on $Z$ and the two-dimensional IR $E^{\prime}$ acts on the (X,Y)components in the $\mathrm{Cu}_{3}$-plane. The only non-zero matrix elements of $\mathbf{R}$ in our basis are $e\left\langle E_{+}^{\prime}, S_{z}\left|X_{-}\right| E_{-}^{\prime}, S_{z}\right\rangle=$ $e\left\langle E_{-}^{\prime}, S_{z}\left|X_{+}\right| E_{+}^{\prime}, S_{z}\right\rangle=2 i d$, with $d$ real denoting the electric dipole coupling, and $X_{ \pm}= \pm X+i Y$ being the irreducible tensor components of $\mathbf{R}$. The zero matrix elements imply that the $S=3 / 2$-states are unaffected in first order in $\mathbf{E}$, and $E_{z}$ has no effect on the spins at all. The resulting coupling between the $\mathbf{E}$-field and chirality is described by $\delta H_{\mathrm{E}}=d \mathbf{E}^{\prime} \cdot \mathcal{C}_{\|}$, where $\mathbf{E}^{\prime}=\mathcal{R}_{z}(\phi) \mathbf{E}$ is rotated by $\phi=7 \pi / 6-2 \theta$ about $z$, and $\mathcal{C}_{\|}=\left(\mathcal{C}_{x}, \mathcal{C}_{y}, 0\right)$.

To emphasize that the spin-electric effect derived above is based on exchange, we reinterpret our results in terms of spin interactions. In an equilateral triangle, and in the absence of electric field, the spin Hamiltonian is given by Eq. (11) with equal exchange couplings $J_{i, i+1} \equiv J$. Using then Eqs. (4) and (5), we find

$$
\delta H_{\mathrm{E}}=\frac{4 d E}{3} \sum_{i=1}^{3} \sin [2(1-i) \pi / 3+\theta] \mathbf{s}_{i} \cdot \mathbf{s}_{i+1},
$$

where $\theta$ is the angle between an in-plane $\mathbf{E}$-field and the vector $\mathbf{r}_{12}$ pointing from site 1 to 2 . This form of $\delta H_{\mathrm{E}}$ shows that the $\mathbf{E}$-field lowers the symmetry by introducing direction-dependent corrections to the exchange couplings $J_{i i+1}$. E.g., if $\theta=\pi / 2, \delta J_{23}=\delta J_{31} \neq \delta J_{12}$. We mention that the eigenstates can be labeled by a partial spin sum quantum number $S_{12}=0,1$ of $\left(\mathbf{s}_{1}+\mathbf{s}_{2}\right)^{2}$.

While the specific form of $d$ in Eq. (7) depends on microscopic details, the structure of $\delta H_{\mathrm{E}}$ relies solely on symmetry arguments and the lack of inversion symmetry is crucial for the spin-electric coupling. Indeed, in inversion-symmetric SMMs, the electric field, which is odd under inversion, and the spin, which is even, can only couple through transitions between orbitals of opposite parity. When the orbital state is quenched, as in the spin Hamiltonian description, there are no orbitals of opposite parity. Thus, in the symmetric case, there is no spin-electric effect up to linear order in $\mathbf{E}$.

Next, we turn to the SOI. The most general form of SOI allowed by the $D_{3 h}$ symmetry reads, $H_{\mathrm{SO}}=\sum_{i=1}^{3}\left[\lambda_{\mathrm{SO}}^{\|} T_{A_{2}^{\prime \prime}} s_{z}^{i}+\lambda_{\mathrm{SO}}^{\perp}\left(T_{E_{+}^{\prime \prime}} s_{-}^{i}+T_{E_{-}^{\prime \prime}} s_{+}^{i}\right)\right]$, where $\lambda_{\mathrm{SO}}^{\perp}\left(\lambda_{\mathrm{SO}}^{\|}\right)$is the effective SOI coupling constant for the $A_{2}^{\prime \prime}-\left(E_{ \pm}^{\prime \prime}\right)$ irreducible representation, and $T_{A_{2}^{\prime \prime}}\left(T_{E_{ \pm}^{\prime \prime}}\right)$ is the corresponding irreducible tensor operator [30]. Using again symmetry group arguments, we find that the SOI Hamiltonian acting in the $S=1 / 2$ subspace reads $\delta H_{\mathrm{SO}}=\Delta_{\mathrm{SO}} \mathcal{C}_{z} S_{z}$, where $\Delta_{\mathrm{SO}}=\lambda_{\mathrm{SO}}^{\|}$. Using Eq. (6), this general form can be reduced to the DzyaloshinskiMoriya interaction given in Eq. (11).

The coupling to a magnetic field $\mathbf{B}$ is given by $\mathbf{B} \cdot \overline{\bar{g}} \cdot \mathbf{S}$, with the Bohr magneton absorbed in the $\overline{\bar{g}}$-tensor. Due to the $D_{3 h}$-symmetry, $\overline{\bar{g}}$ is diagonal with components $g_{x x}=$ $g_{y y}=g_{\perp}$ in the $\mathrm{Cu}_{3}$-plane and $g_{z z}=g_{\|}$normal to it.

Combining $\delta H_{\mathrm{E}}$ and $\delta H_{\mathrm{SO}}$, we finally obtain the effective low-energy Hamiltonian in the presence of SOI and 


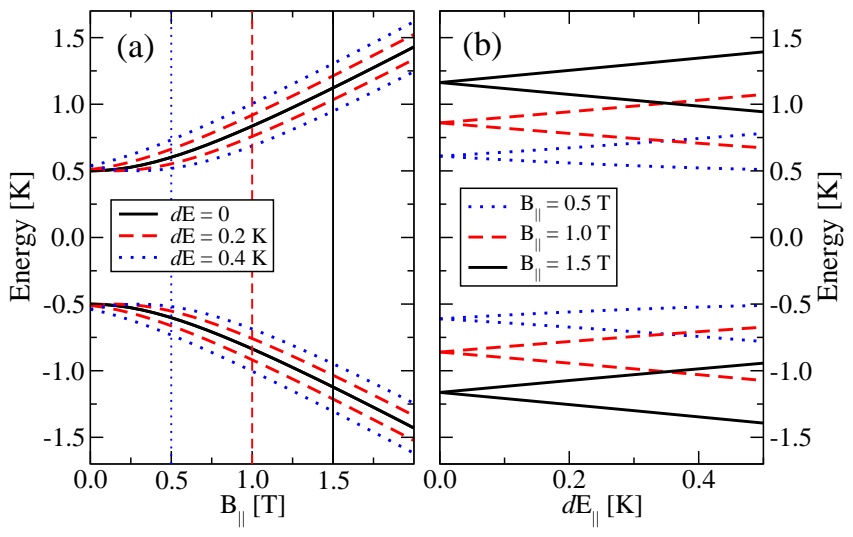

FIG. 2: (Color online) Low-energy spectrum of the $\mathrm{Cu}_{3}$ molecule. (a) Energy levels of the $\mathrm{Cu}_{3}$ molecule in an inplane magnetic field $B_{\|}$(black solid line), split as a static in-plane electric field $E_{\|}$is turned on (dashed red line and dotted blue line). (b) The electric dipole coupling $d$ can be determined from the slope of ESR lines in a constant magnetic field [vertical lines in (a)] as a function of $E_{\|}$.

electric and magnetic fields,

$$
H_{\mathrm{eff}}^{\mathrm{spin}}=\Delta_{\mathrm{SO}} \mathcal{C}_{z} S_{z}+g_{\perp} \mathbf{B}_{\perp} \cdot \mathbf{S}+g_{\|} B_{z} S_{z}+d \mathbf{E}^{\prime} \cdot \mathcal{C}_{\|} \cdot
$$

From this we see that an in-plane E-field causes rotations of the chirality pseudospin. To illustrate the role of $\mathbf{B}$, we focus on the case $\mathbf{E} \| \mathbf{r}_{31}$, corresponding to $\delta H_{\mathrm{E}}=-d E \mathcal{C}_{x}$. For $\mathbf{B} \| z$, the eigenstates coincide with those of $S_{z}$, and thus $\mathbf{E}$ will not induce transitions between $| \pm, 1 / 2\rangle$ and $| \pm,-1 / 2\rangle$, but will do so in subspaces of given $M$, see Fig. 1, For $\mathbf{B} \nVdash z$, instead, the system eigenstates for $\mathbf{E}=0$ are no longer eigenstates of $S_{z}$, and thus the electric field induced transitions result in spin flips, see Fig.1(c).

We emphasize that the electric dipole coupling $d$ in Eq. (8) can be directly accessed in experiments, e.g., by standard ESR measurements [18] in static electric fields, see Fig. 2. While a microscopic evaluation of $d$ requires an ab-initio approach, which is beyond the scope of this work, we can estimate $d,|\mathbf{E}|$ and the spinmanipulation (Rabi) time resulting from Eq. (8) as follows. For $d$ between $d_{\min }=10^{-4} e R_{12}$ and $d_{\max }=e R_{12}$ and for $E \approx 10^{2} \mathrm{kV} / \mathrm{cm}$, obtainable near an STM tip, the Rabi time is $\tau_{\text {Rabi }} \approx 0.1-10^{3} \mathrm{ps}$. The condition $d E \ll \Delta_{\mathrm{H}}$ for the validity of $H_{\mathrm{eff}}^{\mathrm{spin}}$ in Eq. (8) provides another lower bound on the spin-manipulation time, namely $\tau_{\text {Rabi }}^{\min } \approx 10 \mathrm{ps}$.

Hubbard approach. In order to gain further insight into the interplay between the exchange interaction and the electric field, we introduce an $N_{s}$-site Hubbard model of the triangular spin chain. The corresponding Hamiltonian reads, $H_{\mathrm{H}}=$ $\sum_{i, \sigma}\left[\left(U_{i} / 2\right) n_{i, \sigma} n_{i,-\sigma}+\epsilon_{i} n_{i, \sigma}+\left(t_{i i+1} c_{i, \sigma}^{\dagger} c_{i+1, \sigma}+\right.\right.$ h. c. $\left.)\right]$, where $U_{i}$ is the repulsion on site $i, t_{i i+1}$ the hopping
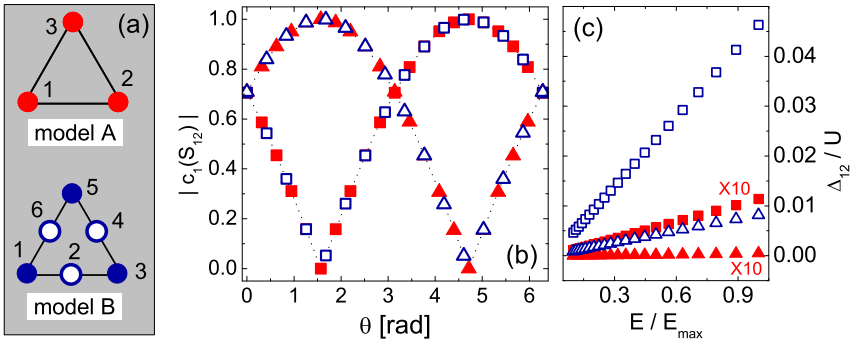

FIG. 3: (Color online) (a) Hubbard models A and B of the spin triangle. Model A: $N_{e}=N_{s}=3, t_{i}=t, U_{i}=U$, and $\epsilon_{i}=$ $\alpha_{i i+1}^{\|}=0$. Model B: $N_{e}=9, N_{s}=6, \alpha_{i i+1}^{\|}=0, \alpha_{i i+1}^{\perp}=\alpha$, $t_{i}=t, \epsilon_{3 k-2}-\epsilon_{3 k^{\prime}-1}=\epsilon, U_{3 k-2}-U_{3 k^{\prime}-1}=U\left(k, k^{\prime}=1,2,3\right)$. (b) Overlap between the projected ground state of $H_{H}+H_{E}$ $\left(\left|\Psi_{1}^{0}\right\rangle\right)$, and the eigenstates of $\mathbf{s}_{1} \cdot \mathbf{s}_{2}$ corresponding to $S_{12}=0$ (squares) and $S_{12}=1$ (triangles), as function of the angle $\theta$ between the triangle side 1-2 and an in-plane $\mathbf{E}$. The filled (empty) symbols correspond to the A (B) model, whereas the dotted lines give the components of the $\delta H_{\mathrm{E}}$ ground state. In both models, $t / U=0.1, e R E / U=2.5 \times 10^{-2}$, and $\alpha=0.1$. (c) Dependence of $\Delta_{21}$ on the amplitude E, for $\mathbf{E} \| y$ and $e R E_{\max } / U=2.5 \times 10^{-2}$. Filled (empty) symbols refer to the A (B) model, squares (triangles) correspond to $\alpha=0.1 \quad(\alpha=0)$ two-site contributions.

matrix element, $\sigma=\uparrow, \downarrow$, and $\sum_{i, \sigma} n_{i}^{\sigma}=N_{\mathrm{e}}$. The coupling of the system to the electric field $\mathbf{E}$ is

$$
H_{\mathrm{E}}=e \mathbf{E} \cdot \sum_{i, \sigma}\left[n_{i, \sigma} \mathbf{r}_{i}+\left(\widetilde{\mathbf{r}}_{i+1} c_{i, \sigma}^{\dagger} c_{i+1, \sigma}+\text { h.c. }\right)\right] .
$$

In the single-site terms, the expectation value of the electron position $\mathbf{r}$ in the Wannier state $\left|\phi_{i}\right\rangle$ is identified with the corresponding ion position, $\left\langle\phi_{i}|\mathbf{r}| \phi_{i}\right\rangle \simeq \mathbf{r}_{i}$. The two-site terms describe the electric-field assisted hopping of electrons between neighboring sites, with $\widetilde{\mathbf{r}}_{i i+1}=$ $\left\langle\phi_{i}|\mathbf{r}| \phi_{i+1}\right\rangle=\alpha_{i i+1}^{\|} \mathbf{r}_{i i+1}+\alpha^{\perp} \mathbf{e}_{z} \times \mathbf{r}_{i i+1}$. We now focus on the two main mechanisms giving antiferromagnetic coupling, namely direct exchange and superexchange (models A and B, Fig. 2(a)). In both cases, the low-energy subspace $\left(\mathcal{S}_{0}\right)$ is defined by the states $\left(\left|\alpha^{0}\right\rangle\right)$ where the magnetic ions at the triangle vertices are singly occupied. For $E=0$, the projection of these states onto $\mathcal{S}_{0}$ $\left(\left|\Psi_{1-8}^{0}\right\rangle\right)$ coincides with the $S=1 / 2$ and $S=3 / 2$ eigenstates of the Heisenberg Hamiltonian. The degeneracy in the $S=1 / 2$ multiplet is lifted by an electric field.

In Fig. [3(b), we show the overlap between the projected ground state $\left|\Psi_{1}^{0}\right\rangle$ and the $\left|S=1 / 2, S_{12}=0,1\right\rangle$ states for a given $S_{z}$ as function of the direction of $\mathbf{E}$ (angle $\theta$ ). The results coincide with the ones from Eq. (7), for both models $\mathrm{A}$ and $\mathrm{B}$. In addition, we find that the splitting $\left(\Delta_{21} \equiv E_{2}-E_{1}\right)$ between the two lowest energies varies by less than $5 \%$ with $\theta$, in agreement with $H_{\mathrm{E}}^{\text {spin }}$ that predicts no $\theta$-dependence at all.

In Fig. [3(c) we isolate the contribution to $\Delta_{21}$ arising 
from the single- and two-site terms. All these contributions scale linearly with $\mathbf{E}$ for every $\theta$. The dependence of $\Delta_{21}$ on $t$, however, is model-dependent. In particular, in model $\mathrm{A}$, the contributions to $\Delta_{21}$ arising from the single- and two-site terms scale like $(t / U)^{3}$ and $(t / U)$, resp. Analogous power-law dependences are found in model $\mathrm{B}$, where the single-site (two-site) contribution scales as $(t / U)^{4}\left((t / U)^{3}\right)$, and two-site terms dominate in both models when $t \ll U$. Additional mechanisms, such as the relative displacements of the ions, can contribute to the coupling between spin and electric field.

Spin coupling to cavity electric fields. Exchange coupling of SMMs has been demonstrated in dimers [31]. The use of this short-range and (so far) untunable interaction requires additional resources for quantum information processing [13]. Efficient spin-electric interaction, on the other hand, provides a route to long-range and switchable coupling between SMM qubits. In particular, microwave cavities are suitable for reaching the strongcoupling regime for various qubit systems [22, 23, 24, 25]. Here, we propose to use such cavities to control single SMMs and, moreover, to couple the spin-qubits of distant SMMs placed inside the same cavity.

The interaction of a single SMM with the cavity field reads, $\delta H_{\mathrm{E}}=d \mathbf{E}_{0}^{\prime} \cdot \mathbf{C}_{\|}\left(b^{\dagger}+b\right)$, where $\mathbf{E}_{0}^{\prime}$ is the rotated electric field of amplitude $\left|\mathbf{E}_{0}\right| \propto \sqrt{\hbar \omega / \mathcal{V}}$ inside the cavity of volume $\mathcal{V}$ [23], and $b$ is the annihilation operator for the photon mode of frequency $\omega$. In the rotating wave approximation, the low-energy Hamiltonian of $N$ SMMs interacting with the cavity mode is $H_{\mathrm{s}-\mathrm{ph}} \equiv \sum_{j} H^{(j)}+\omega b^{\dagger} b$, where

$H^{(j)}=\Delta_{\mathrm{SO}} \mathcal{C}_{z}^{(j)} S_{z}^{(j)}+\mathbf{B} \cdot \overline{\bar{g}} \cdot \mathbf{S}^{(j)}+d E_{0}\left(e^{i \varphi_{j}} b^{\dagger} \mathcal{C}_{-}^{(j)}+\right.$ h. c. $)$

with $\mathcal{C}_{ \pm}^{(j)}=\mathcal{C}_{x}^{(j)} \pm i \mathcal{C}_{y}^{(j)}$ and $\varphi_{j}=\theta_{j}+7 \pi / 6$. Here, $H_{\mathrm{s}-\text { oh }}$ reduces to the well-known Tavis-Cummings model 32] when the spins are in eigenstates of $S_{z}^{(i)}$, and $\mathbf{B} \| z$. However, if $\mathbf{B} \nVdash z$ it is possible to couple both chiralities and total spins of distant molecules. Typically, the electric fields in cavities are weaker, $\left|\mathbf{E}_{0}\right| \approx 1 \mathrm{~V} / \mathrm{cm}$ for $\hbar \omega \approx 0.1 \mathrm{meV}[25]$, than the ones near STM tips, thus giving $\tau_{\text {Rabi }} \approx 0.01-100 \mu \mathrm{s}$. Obviously, decreasing the cavity volume $\mathcal{V}$ would give shorter $\tau_{\text {Rabi }}$. Coupling of distant SMMs can be controlled by tuning two given molecules in and out of resonance with the cavity mode, e.g., by applying additional local electric fields. Further effects such as the state transfer between stationary and flying qubits, or the SMM-photon entanglement, can be observed in a system described by $H_{\mathrm{s}-\mathrm{ph}}$.

In conclusion, we find an exchange-based mechanism that couples electric fields to spins in triangular molecular antiferromagnets. While our results are derived for $\mathrm{Cu}_{3}$, analogous symmetry arguments are expected to apply to other molecular magnets that lack inversion symmetry, such as $\mathrm{V}_{15}$ [33], $\mathrm{Co}_{3}$ [34], $\mathrm{Dy}_{3}$ [35], $\mathrm{Mn}_{12}[2$, 3, 4] , etc.
We thank D. Klauser, M. Affronte and V. Bellini for useful discussions. We acknowledge financial support from the Swiss NSF, the NCCR Nanoscience Basel; the Italian MIUR under FIRB Contract No. RBIN01EY74; the EU under "MagMaNet" and "QuEMolNa".

[1] D. Gatteschi, R. Sessoli, and J. Villain, Molecular Nanomagnets (Oxford Univ. Press, New York 2007).

[2] J. R. Friedman, M. P. Sarachik, J. Tejada, and R. Ziolo, Phys. Rev. Lett. 76, 3830 (1996).

[3] L. Thomas, F. Lionti, R. Ballou, D. Gatteschi, R. Sessoli, and B. Barbara, Nature 383, 145 (1996).

[4] L. Bokacheva, A. D. Kent, and M. A. Walters, Phys. Rev. Lett. 85, 4803 (2000).

[5] C. Romeike, M. R. Wegewijs, W. Hofstetter, and H. Schoeller, Phys. Rev. Lett. 96, 196601 (2006).

[6] M. N. Leuenberger and E. R. Mucciolo, Phys. Rev. Lett. 97, 126601 (2006).

[7] J. Lehmann and D. Loss, Phys. Rev. Lett. 98, 117203 (2007).

[8] A. Ardavan, O. Rival, J. J. L. Morton, S. J. Blundell, A. M. Tyryshkin, G. A. Timco, and R. E. P. Winpenny, Phys. Rev. Lett. 98, (2007).

[9] M. N. Leuenberger and D. Loss, Nature 410, 789 (2001).

[10] F. Meier, J. Levy, and D. Loss, Phys. Rev. B 68, 134417 (2003).

[11] F. Troiani, A. Ghirri, M. Affronte, S. Carretta, P. Santini, G. Amoretti, S. Piligkos, G. Timco, and R. Winpenny, Phys. Rev. Lett. 94, 207208 (2005).

[12] J. Lehmann, A. Gaita-Ariño, E. Coronado, and D. Loss, Nature Nanotechnology 2, 312 (2007).

[13] F. Troiani, M. Affronte, S. Carretta, P. Santini, and G. Amoretti, Phys. Rev. Lett. 94, 190501 (2005).

[14] M. Affronte, I. Casson, M. Evangelisti, A. Candini, S. Carretta, C. Muryn, S. Teat, G. Timco, W. Wernsdorfer, and R. Winpenny, Angew. Chem. Int. Ed. 44, 6496 (2005).

[15] L. Bogania and W. Wernsdorfer, Nature Mater. 7, 179 (2008).

[16] S. Bertaina, S. Gambarelli, A. Tkachuk, I. N. Kurkin, B. Malkin, A. Stepanov, and B. Barbara, Nature Nanotechnology 2, 39 (2007).

[17] M. Bal, J. R. Friedman, W. Chen, M. T. Tuominen, C. C. Beedle, E. M. Rumberger, and D. N. Hendrickson, Europhys. Lett. 82, 17005 (2008).

[18] A. Bencini and D. Gatteschi, EPR of Exchange Coupled Systems (Springer-Verlag, Berlin Heidelberg, 1989).

[19] W. A. Hofer, A. S. Foster, and A. L. Shluger, Rev. Mod. Phys. 75, 1287 (2003).

[20] C. F. Hirjibehedin, C. P. Lutz, and A. J. Heinrich, Science 312, 1021 (2006).

[21] A. C. Bleszynski, L. Fröberg, M. T. Björk, H. J. Trodahl, L. Samuelson, and R. M. Westervelt, arXiv:0802.3236 (2008).

[22] A. Wallraff, D. I. Schuster, A. Blais, L. Frunzio, R.-S. Huang, J. Majer, S. Kumar, S. M. Girvin, and R. J. Schoelkopf, Nature 431, 162 (2004).

[23] G. Burkard and A. Imamoglu, Phys. Rev. B 74, 041307 (2006).

[24] A. André, D. DeMille, J. M. Doyle, M. D. Lukin, S. E. 
Maxwell, P. Rabl, R. J. Schoelkopf, and P. Zoller, Nature Physics 2, 636 (2006).

[25] M. Trif, V. N. Golovach, and D. Loss, Phys. Rev. B 77, 045434 (2008).

[26] E. I. Rashba and A. L. Efros, Phys. Rev. Lett. 91, 126405 (2003).

[27] V. N. Golovach, M. Borhani, and D. Loss, Phys. Rev. B 74, 165319 (2006).

[28] K. C. Nowack, F. H. L. Koppens, Y. V. Nazarov, and L. M. K. Vandersypen, Science 318, 1430 (2007).

[29] K.-Y. Choi, Y. H. Matsuda, H. Nojiri, F. Hussain, A. C. Stowe, C. Ramsey, and N. S. Dalal, Phys. Rev. Lett. 96, 107202 (2006).

[30] B. Tsukerblat, Group theory in chemistry and spec- troscopy (Academic Press, New York, 1994).

[31] W. Wernsdorfer, N. Allaga-Alcalde, D. N. Hendrickson, and G. Christou, Nature 416, 406 (2002).

[32] S. Haroche and J.-M. Raimond, Exploring the Quantum: Atoms, Cavities, and Photons (Oxford University Press, New York, 2006).

[33] I. Chiorescu, W. Wernsdorfer, A. Müller, H. Bögge, and B. Barbara, Phys. Rev. Lett. 84, 3454 (2000).

[34] M. C. Juan, E. Coronado, A. G. Arino, C. G. Saiz, H.U. Güdel, A. Sieber, R. Bircher, and H. Mutka, Inorg. Chem. 44, 3389 (2005).

[35] J. Luzon, K. Bernot, I. J. Hewitt, C. E. Anson, A. K. Powell, and R. Sessoli, arXiv:0804.1272 (2008). 